\title{
An Investigation to Study the Role of Novel Rhenium Compounds on Endometrial Uterine Cancer Cell Lines
}

\author{
Christopher Krauss ${ }^{1}$, Chelsey Aurelius ${ }^{2}$, Kayla Johnston ${ }^{2}$, Victoria Bartlette ${ }^{2}$, Sherrita \\ Gavin $^{2}$, Jazmine Cuffee ${ }^{2}$, Satyendra Banerjee ${ }^{2}$, Sarah Wiseniewsky², Santosh Mandal ${ }^{1, *}$ \\ and Hirendra Nath Banerjee ${ }^{2, *}$ \\ ${ }^{1}$ Department of Chemistry, Morgan State University, Baltimore, MD 21251, USA \\ ${ }^{2}$ Department of Natural, Pharmacy and Health Sciences, Elizabeth City State University Campus of the \\ University of North Carolina, Elizabeth City, NC 27909, USA
}

\begin{abstract}
Endometrial cancer of the uterus is highly malignant with an increase rate of morbidity and mortality in both childbearing age and postmenopausal women. Rhenium compounds have been shown to have therapeutic properties against various cancers both in vitro cell lines and in vivo animal models. In this in vitro study, we investigated the effects of a novel group of Rhenium ligands on a uterine cancer cell line. Our initial results showed that these compounds are cytotoxic, induces apoptosis and prevents tubulin polymerization in these uterine cancer cell lines, we also found these novel Rhenium compounds to be noncytocidal to healthy human blood lymphocyte cells, thus proving their safety and efficacy in future translational studies.
\end{abstract}

Keywords: Rhenium Compounds, Uterine Cancer, Apoptosis, Tubulin, lymphocytes.

\section{INTRODUCTION}

Despite ongoing research, cancer remains a deadly disease that impacts millions of patients. Platinum based treatment options have been effective in the past, but there are limitations to these compounds [1]. and certain cancers are becoming resistant to treatment with platinum-based compounds [find citation]. To help overcome the limitations and resistance to platinum-based therapies, alternatives to platinum have been studied in recent years [2]. Rhenium-based compounds are an appealing alternative to platinum-based compounds. These rhenium-based compounds are typically less toxic to healthy tissue than platinum-based compounds, while also providing anti-cancer properties [3].

Endometrial cancer is the most commonly diagnosed gynecologic cancer. About 50,000 American women are diagnosed with the disease every year [4]. Endometrial cancer is also the most common form of uterine cancer, so it is frequently referred to as uterine cancer [5]. Cisplatin and other platinum compounds are effective in Endometrial cancer treatment but toxicity of these drugs to the systemic organs calls for searching safer effective alternative chemotherapeutic agents [6].

*Address correspondence to these authors at the Department of Chemistry Morgan State University, Baltimore, MD 21251, USA; E-mail: santosh.mandal@morgan.edu

Department of Natural, Pharmacy and Health Sciences, Elizabeth City State University Campus of the University of North Carolina, Elizabeth City, NC 27909, USA; E-mail: bhirendranath@ecsu.edu
In this study, rhenium-based compounds were synthesized and tested against an endometrial cancer cell line as well as a healthy lymphocyte cell line for toxicity studies. Data was collected to observe toxicity and anti-cancer properties; inducing apoptosis and interactions with tubulin.

\section{MATERIALS AND METHODS}

Rhenium compounds were synthesized with different ligands according to the procedure described in our earlier study [5] and named PR series for the following drugs:

PR-1: Tricarbonylperrhenato(bpy)rhenium(I)

PR-2: Tricarbonylperrhenato(Phen)rhenium(I)

PR-3: Tricarbonylperrhenato(5-MePhen)rhenium(I)

PR-4: Tricarbonylperrhenato(neocuproin)rhenium(I)

PR-5: Tricarbonylperrhenato(5,6- $\left.\mathrm{Me}_{2} \mathrm{Phen}\right)$ rhenium(I)

PR-6: Tricarbonylperrhenato(bathophen)rhenium(I)

PR-7: Tricarbonylperrhenato(bathocuproine)rhenium(I)

PR-8: Tricarbonylperrhenato(4,7- $\mathrm{Me}_{2}$ Phen)rhenium(I)

PR-9:

Tricarbonylperrhenato $(3,4,7,8-$

$\mathrm{Me}_{4}$ Phen)rhenium(I)

An additional highly effective Rhenium compound was also synthesized named as

$\mathrm{CH}-2$ : Tricarbonylchloro(bpy)rhenium(I) 
All the drugs were dissolved in DMSO at different concentrations which was also used as a vehicular control.

\section{Cell Culture}

HTB112 endometrial cancer cell lines were obtained from ATCC (USA) and cultured in McCoy's medium supplemented with FBS, Penicillin and Streptomycin and maintained at $37 \mathrm{C}$ in $5 \% \mathrm{CO} 2$ incubator.

The healthy lymphocyte cell lines were obtained from Coriell Institute (USA) and cultured in RPMI medium supplemented with FBS, Penicillin and Streptomycin and maintained at $37 \mathrm{C}$ in $5 \% \mathrm{CO} 2$ incubator.

\section{LDH Assay}

Lactate Dehydrogenase assay was done by using a LDH assay kit from Abcam Corporation (USA) following standard protocol using a microplate reader at $405 \mathrm{~nm}$ to study cytotoxicity of the PR series 1-9 Rhenium compounds at 2 micromolar dose with DMSO as vehicular control.

\section{MTT Assay}

MTT assay reagents were purchased from Fisher Scientific Corporation (USA) and the assay was performed on $\mathrm{CH}$-treated healthy human lymphocytes incubated for 48 hours at various concentrations. The results were determined using a standard plate reader.

\section{Apoptosis Assay}

Annexin V-APC Assay Kit from Abcam Corporation (USA) employs an APC-conjugated Annexin $\mathrm{V}$ as a probe for phosphatidylserine detection on the outer membrane of apoptotic cells; After treating the HTB112 cell lines with the $\mathrm{CH} 2$ compound at 2 micromolar dose with DMSO vehicular control for 48 hours, the following steps were carried out. First, we collected 1-5 x 105 cells by centrifugation. Next the cells were resuspended in $500 \mu \mathrm{L}$ of $1 \mathrm{X}$ binding buffer, $5 \mu \mathrm{L}$ of annexin V-FITC was added and subsequently Incubated at room temperature for $5 \mathrm{~min}$ in the dark. Photographs were finally taken under a fluorescence microscope.

\section{Tubulin Assay}

Tubulin Tracker Deep Red provides deep-red/farred fluorescence when bound to polymerized tubulin in living cells. Tubulin Tracker Deep Red is based on Docetaxel conjugated with a bright, photostable deepred fluorophore. Docetaxel belongs to the family of cytoskeletal drugs that target tubulin. Tubulin Tracker Deep Red absorbs and emits optimally at $652 \mathrm{~nm}$ and $669 \mathrm{~nm}$, respectively and can be visualized with standard Cy5 filter settings using almost any fluorescent imaging instrument. After 48 hours of incubation, $\mathrm{CH} 2$ treated HTB112 cells-at $2 \mu \mathrm{M}$ dose and DMSO control- were stained by Tubulin tracker red dye purchased from Fisher Scientific Corporation (USA) and photographed under fluorescence microscope.

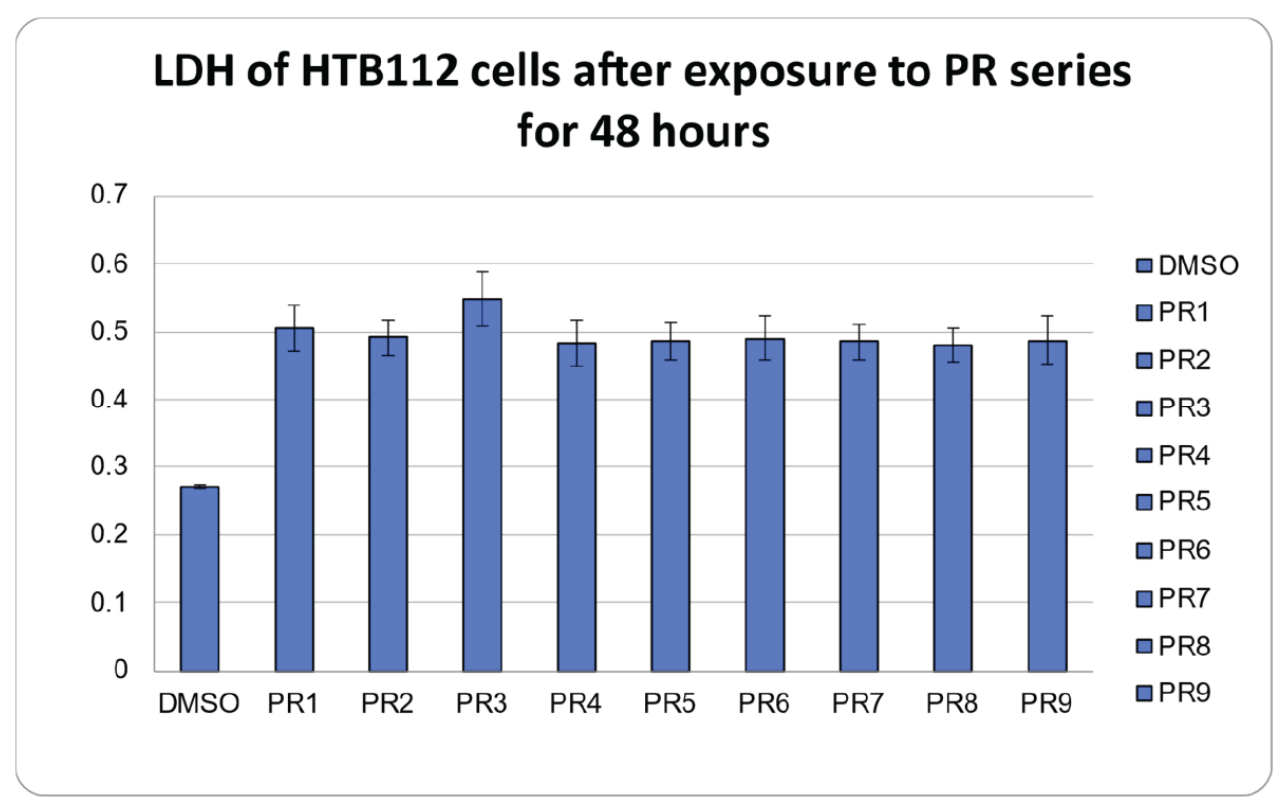

Figure 1: Data showing the cytotoxic effects of the novel PR series compounds at a dose of $2 \mathrm{uM}$ on the uterine cancer cell lines exposed for 48 hours, DMSO was used as vehicular control for each experiment. 


\section{RESULTS}

We observed effective cytotoxicity of the PR series compounds on uterine cancer cell lines at a minimal dose of $2 u m$ at 48 hours incubation (Figure 1), however, the ongoing COVID-19 Pandemic caused the lack of chemical supplies and we continued our research work to find out the effectiveness of our other
Rhenium ligand compound, $\mathrm{CH} 2$. We initially tested the toxic effects of this Rhenium compound on a healthy human Lymphocyte cell line by MTT assay. Our results showed that at 2 um dose, the drug did not induce significant cell death to these cells supporting the safety of this compound (Figure 2). Next, an apoptosis assay with Annexin $\mathrm{V}$ assay kit was carried out and it was found that $\mathrm{CH} 2$ compound induces apoptosis of

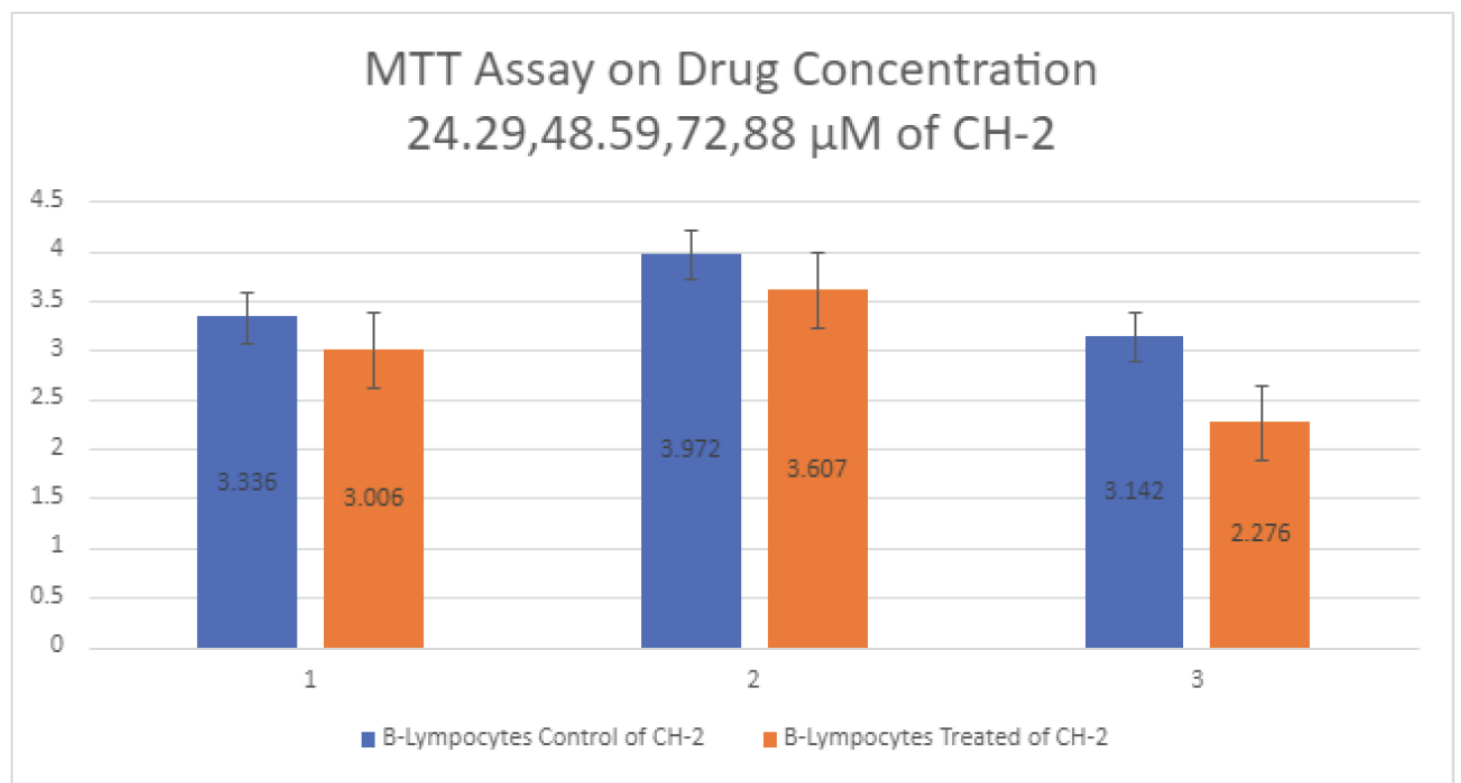

Figure 2: MTT studies on B-lymphocytes showed that $\mathrm{CH}-2$ has low toxicity to human lymphocyte cells. By adding different concentrations of $\mathrm{CH} 2$ along with vehicular control DMSO to cells for 48 hours, there was little difference in cell death observed in the treated compared to the control.

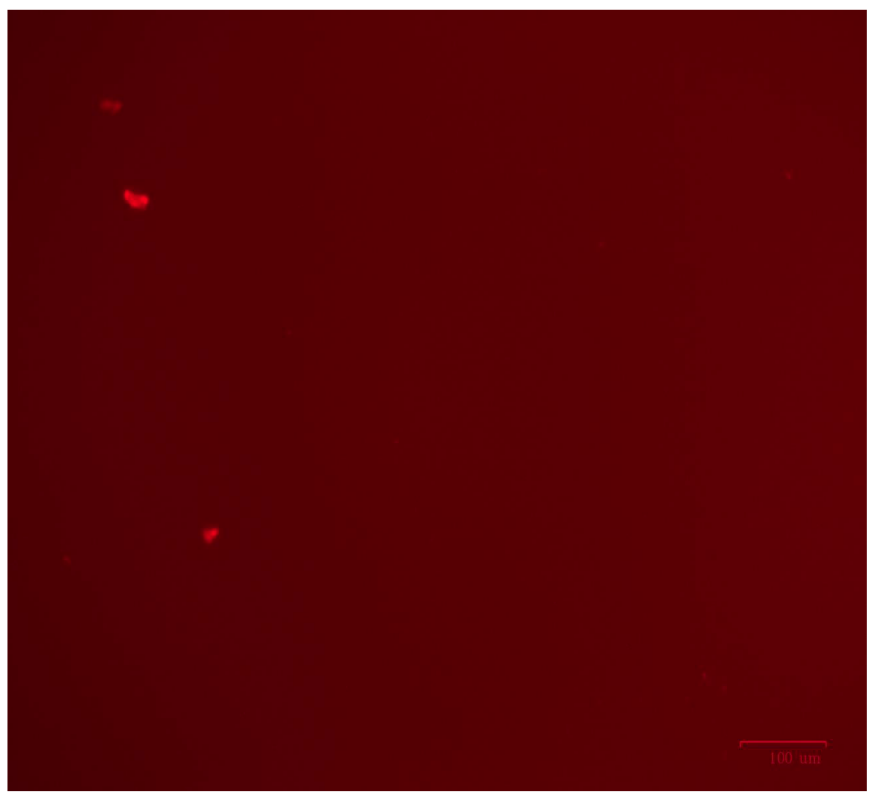

a

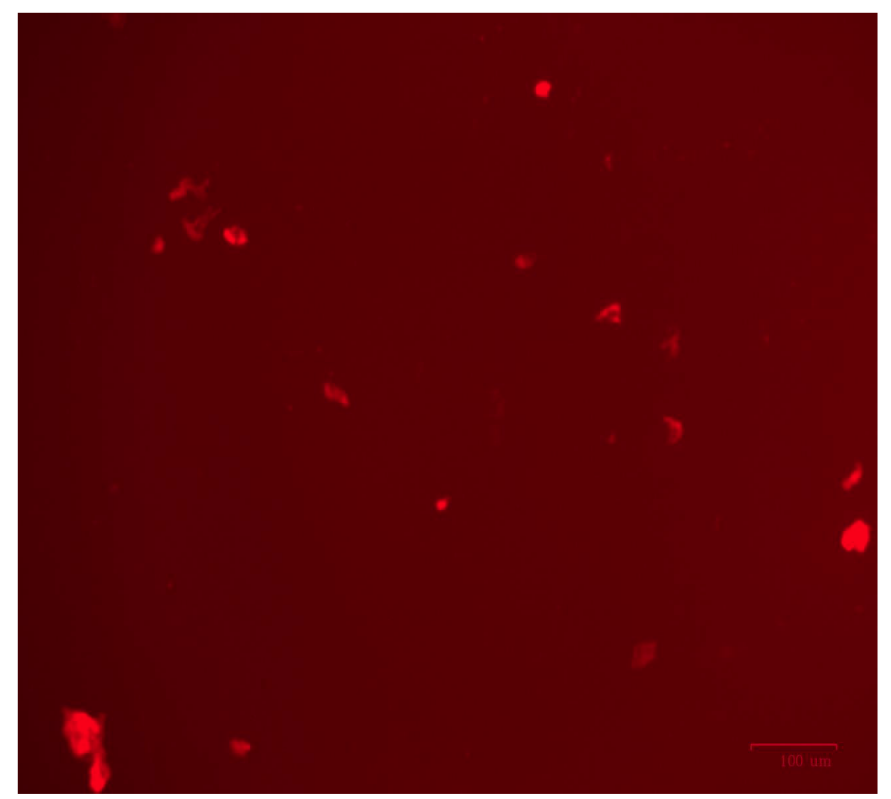

b

Figure 3: $\mathbf{a}$ and b: By using Tubulin Tracker Deep Red to measure tubulin in cells, changes in tubulin were measured after exposure to $\mathrm{CH} 2$ compound. More tubulin was observed in cells exposed to the $\mathrm{CH} 2$ than in control cells exposed only to DMSO. This suggests that the $\mathrm{CH}$ series of rhenium-based compounds may provide their anti-cancer properties by targeting tubulin. 


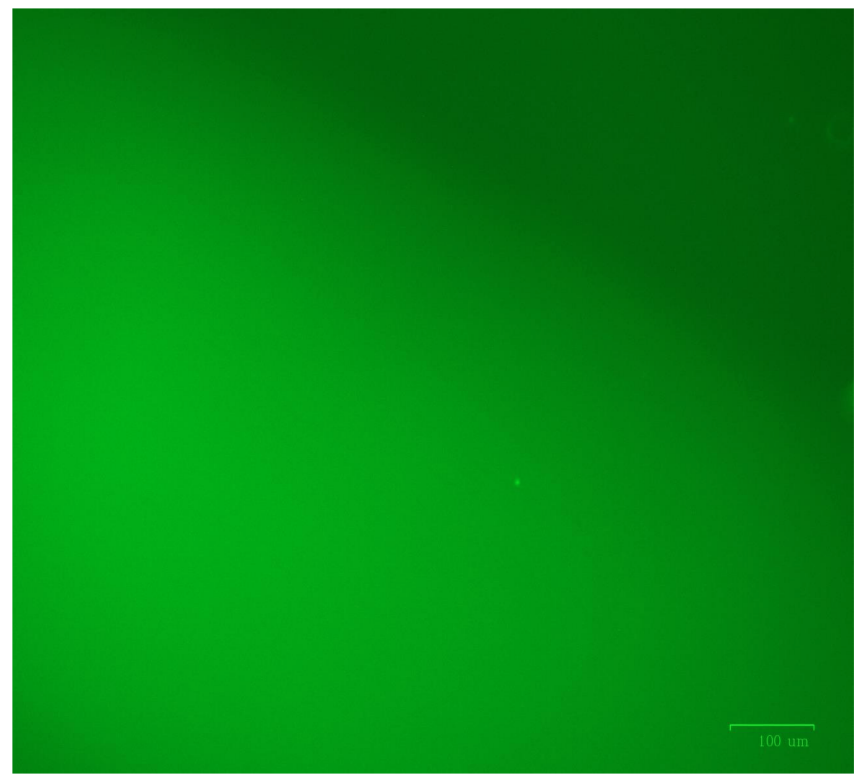

a

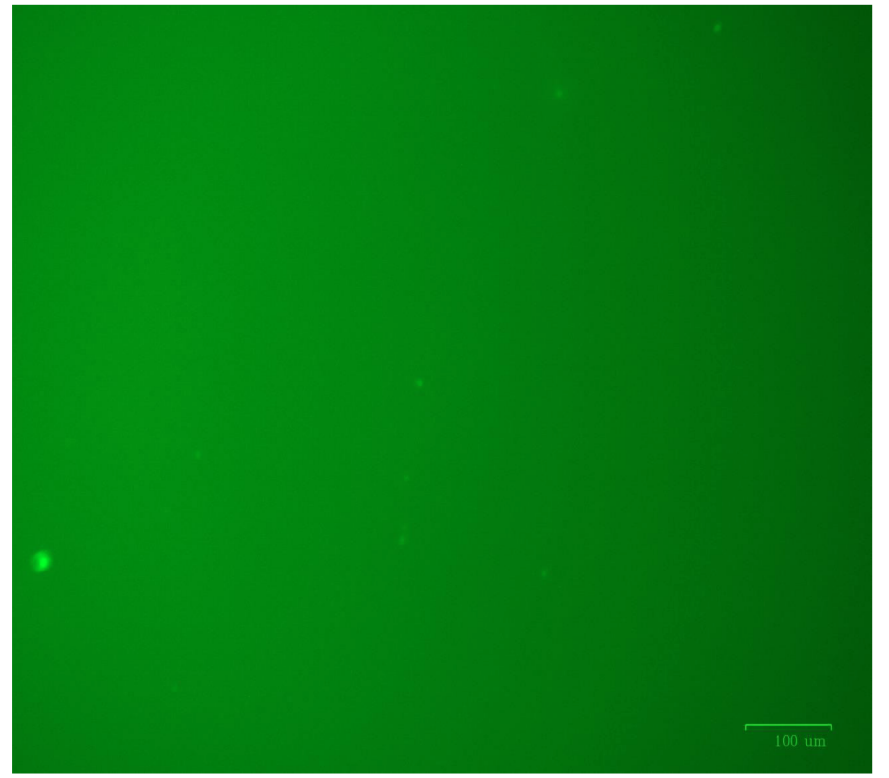

b

Figure 4: a. Annexin $\mathrm{V}$ apoptosis detection of Vehicular control DMSO treated Uterine cancer cells. b: Using the annexin $\mathrm{V}$ FITC apoptosis detection kit, an increase in apoptosis was detected in Uterine cancer cells exposed to $\mathrm{CH}-2$ Figure $\mathbf{4 b}$ in comparison to DMSO vehicular control for 48 hours.

the HTB112 endometrial cancer cell lines (Figure 3). In order to further investigate the precise mechanism of cell death induced by this drug, a Tubulin tracker assay was performed and $\mathrm{CH} 2$ Rhenium compound was found to prevent tubulin aggregation which could be a possible cause of cell death (Figure 4).

\section{DISCUSSION}

Endometrial cancer has a very high incidence among women of child bearing age due to hormonal influence, it causes a high rate of mortality and morbidity [12-14]. Though Cisplatin and Carboplatin are effective, they produce highly toxic adverse effects. This study was aimed at searching for an alternative drug in the form of different Rhenium ligands. Earlier in vitro and vivo studies, including some from our lab, have reported that Rhenium compounds show anticancer properties against many types of neoplasms $[2,6,11]$. The Rhenium compounds used in this study were named as the PR series and $\mathrm{CH} 2$ compound. Although, the PR series compounds showed effective anticancer properties against uterine cancer cells, as evidenced by our LDH assays, the unavailability of reagents due to current pandemic compelled us to continue with the $\mathrm{CH} 2$ Rhenium compound. The $\mathrm{CH} 2$ Rhenium ligand not only demonstrated anticancer efficacy, as evidenced by the apoptosis and anti tubulin assay, but also showed lack of toxicity to the healthy lymphocyte cells even at a very high dose, unlike the platinum based anticancer drugs which are extremely toxic to blood cells causing anemia and susceptibility to infections as their side effects. This is the first study to reveal that the Rhenium compound, $\mathrm{CH} 2$, is capable of preventing tubulin polymerization playing a possible role in blocking cell division and proliferation, hopefully will be investigated on other Rhenium ligands in the future. We also found that the nature of cell death initiated by these Rhenium compounds was through the apoptotic pathway instead of necrosis. Further studies to investigate the role of these novel Rhenium compounds in other types of cancers and to study tubulin polymerization preventing properties of other Rhenium ligands may prove helpful in transferring them from bench to bedside as an effective chemotherapeutic agent.

\section{ACKNOWLEDGEMENT}

This work is supported by the NIH-MARC award, grant\# 2T34GM2300831-07.

\section{REFERENCES:}

[1] Collery P, Desmaele D, Vijaykumar V. Design of Rhenium Compounds in Targeted Anticancer Therapeutics. Curr Pharm Des 2019; 25(31): 3306-3322.

https://doi.org/10.2174/1381612825666190902161400

[2] Parson C, Smith V, Krauss C, Banerjee HN, Reilly C, Krause $\mathrm{JA}$, et al. Anticancer Properties of Novel Rhenium Pentylcarbanato Compounds against MDA-MB-468(HTB132) Triple Node Negative Human Breast Cancer Cell Lines. Br J Pharm Res 2015; 4(3): 362-367. https://doi.org/10.9734/BJPR/2014/4697

[3] Knopf KM, Murphy BL, MacMillan SN, Baskin JM, Barr MP, Boros $\mathrm{E}$, et al. In vitro Anticancer Activity and in vivo 
Biodistribution of Rhenium(I) Tricarbonyl Aqua Complexes. J Am Chem Soc 2017; 139(40): 14302-14314. https://doi.org/10.1021/jacs.7b08640

[4] Braun MM, Overbeek-Wager EA, Grumbo RJ. Diagnosis and Management of Endometrial Cancer. Am Fam Physician 2016; 93(6): 468-74.

[5] Moore K, Brewer MA. Endometrial Cancer: Is This a New Disease? Am Soc Clin Oncol Educ Book 2017; 37: 435-442. https://doi.org/10.14694/EDBK_175666

[6] Wilder PT, Weber DJ, Winstead A, Parnell S, Hinton TV, Stevenson $\mathrm{M}$, et al. Unprecedented anticancer activities of organorhenium sulfonato and carboxylato complexes against hormone-dependent MCF-7 and hormone-independent triplenegative MDA-MB-231 breast cancer cells. Mol Cell Biochem 2018; 441(1-2): 151-163. https://doi.org/10.1007/s11010-017-3181-z

[7] Yang J, Zhao JX, Cao Q, Hao L, Zhou D, Gan Z, et al. Simultaneously Inducing and Tracking Cancer Cell Metabolism Repression by Mitochondria-Immobilized Rhenium(I) Complex. ACS Appl Mater Interfaces 2017; 9(16): 13900-13912.

https://doi.org/10.1021/acsami.7b01764

[8] Estrada LD, Duran E, Cisterna M, Echeverria C, Zheng Z, Borgna $\mathrm{V}$, et al. The cluster [Re6Se8I6]3- penetrates biological membranes: drug-like properties for CNS tumor treatment and diagnosis. Biometals 2018; 31(4): 517-525. https://doi.org/10.1007/s10534-018-0097-3

[9] Martínez-Lillo J, Mastropietro TF, Lappano R, Madeo A, Alberto ME, Russo $\mathrm{N}$, et al. Rhenium(IV) compounds inducing apoptosis in cancer cells. Chem Commun (Camb) 2011; 47(18): 5283-5. https://doi.org/10.1039/c1cc11038a

[10] Yang J, Zhao JX, Cao Q, Hao L, Zhou D, Gan Z, et al. Simultaneously Inducing and Tracking Cancer Cell Metabolism Repression by Mitochondria-Immobilized Rhenium(I) Complex. ACS Appl Mater Interfaces 2017; 9(16): 13900-13912. https://doi.org/10.1021/acsami.7b01764

[11] Parson C, Smith V, Krauss C, Banerjee HN, Reilly C, Krause $\mathrm{JA}$, et al. The effect of novel rhenium compounds on lymphosarcoma, PC-3 prostate and myeloid leukemia cancer cell lines and an investigation on the DNA binding properties of one of these compounds through electronic spectroscopy. J Bioprocess Biotech 2013; 4(1): 141.

[12] Henley SJ, Miller JW, Dowling NF, Benard VB, Richardson LC. Uterine Cancer Incidence and Mortality - United States, 1999-2016. MMWR Morb Mortal Wkly Rep 2018; 67(48): 1333-1338. https://doi.org/10.15585/mmwr.mm6748a1

[13] Kravchenko J, Akushevich I, Rhew SH, Agarwal P, Lyerly HK. Uterine Cancer Mortality in White and African American Females in Southeastern North Carolina. J Environ Public Health 2020; 2020: 6734031. https://doi.org/10.1155/2020/6734031

[14] Jones NL, Huang Y, Chatterjee S, Tergas Al, Burke WM, Hou JY, et al. Patterns of care and outcomes for women with uterine cancer and ovarian metastases. Int J Gynecol Cancer 2019; 29(2): 365-376. https://doi.org/10.1136/ijgc-2018-000047

\section{DOI: https://doi.org/10.30683/1929-2279.2020.09.12}

\section{(C) 2020 Krauss et al.; Licensee Neoplasia Research.}

This is an open access article licensed under the terms of the Creative Commons Attribution Non-Commercial License (http://creativecommons.org/licenses/by-nc/3.0/) which permits unrestricted, non-commercial use, distribution and reproduction in any medium, provided the work is properly cited. 